\title{
Medición de la diversidad comercial minorista en áreas urbanas a través del uso de los índices de Shannon-Weaver y de Ullman-Dacey ${ }^{1}$ \\ Measurement of diversity of commercial retail in urban areas through use of Shannon- Weaver and Ullman and Dacey indexes
}

\author{
Antonio Aguilera Ontiveros² y Mayra Angélica Bárcenas Castro ${ }^{3}$
}

\section{INTRODUCCIÓN}

La diversidad es una variable relacionada con la estabilidad económica de los sistemas urbanos y regionales. Es ampliamente aceptada la tesis de que a mayor diversidad de las unidades económicas en una ciudad, la economía de ésta puede soportar cambios cíclicos en la demanda y por ende el empleo global se mantiene en límites aceptables (Bahl, Firestine y Phares, 1971; Simon, 1988; Trendle y Shorney, 2003; Izraeli y Murphy 2003; Mason y Howard, 2010). De hecho, Simon (1988) e Izraeli y Murphy (2003) han mostrado, para casos específicos, que la tasa de desempleo es menor entre más diversificada industrialmente sea la ciudad.

Dos maneras de medir la diversidad industrial son el índice de diversidad de Shannon-Weaver (Attaran, y Zwick, 1987a; 1987b; Nissan y Carter, 2010)

\footnotetext{
${ }^{1}$ Este trabajo fue parcialmente financiado por el Consejo Nacional de Ciencia y Tecnología de México a través del proyecto $\mathrm{n}^{\circ}$. 101366 . Nuestro agradecimiento.

${ }^{2}$ El Colegio de San Luis, A.C. Programa de Estudios Políticos e Internacionales. aaguilera@colsan.edu.mx

${ }^{3}$ El Colegio de San Luis, A.C. Programa de Estudios Políticos e Internacionales.
} 
y el índice de Ullman-Dacey (Ullman y Dacey, 1960; Bahl, Firestine y Phares, 1971; Brodsky y Sarfaty, 1977, Grossberg, 1980).

En este artículo aplicamos los índices de Shannon-Weaver y Ullman-Dacey para indagar la diversidad comercial minorista de los dos principales centros de actividad económica de la zona metropolitana de San Luis Potosí, México. Para esta zona metropolitana, el comercio al por menor es una actividad económica que genera el 56.01\% de los empleos dentro de la urbe (INEGI, 2012b), lo que hace relevante su análisis. Además, son realmente nulos los estudios al respecto para esta ciudad, lo que implica que se está generando nuevo conocimiento útil localmente.

En la primera parte de este trabajo se establecen los formalismos matemáticos de los índices de Shannon-Weaver y de Ullman-Dacey. Enseguida se muestra la forma en que se realizó la definición del área de estudio, el procedimiento de obtención de los datos, el modo en que se trabajaron dichos datos para usarlos en los cálculos para cada índice. Por último, se realiza una discusión sobre los resultados y se hacen reflexiones en materia de planeación urbana y económica para la ciudad en cuestión.

\section{El ÍNDice de Shannon-Weaver y el índice de Ullman-Dacey}

El índice de Shannon-Weaver (Dickman, 1968), también conocido como entropía informática (Shannon, 1948), mide la información de un sistema. Shannon estableció el índice para medir la incertidumbre relacionada con «adivinar» una cadena de texto, dado que se conocen algunas letras iniciales. La idea básica es que entre mayor número de letras diferentes exista en la cadena, con respecto a su abundancia proporcional, más difícil es predecir correctamente qué letra será la siguiente en la cadena. La entropía de Shannon cuantifica la incertidumbre o entropía asociada con dicha predicción.

Para el caso que nos ocupa el índice mide la diversidad con respecto a unidades económicas de un área urbana (Attaran, y Zwick, 1987a; 1987b; Nissan y Carter, 2010). Usando el índice se puede calcular la diversidad para cada una de las áreas en que la ciudad ha sido segmentada con fines censales o bien se puede llevar a más nivel de detalle, analizando las manzanas que contiene un área censal.

El índice se calcula de la siguiente forma:

$$
H=-\sum_{i=1}^{n} p_{i} \log _{2} p_{i}
$$


Dónde $p_{\mathrm{i}}$ es la probabilidad de la i-ésima actividad económica en el área bajo estudio, esto es, sí se tienen $n$ unidades económicas de la i-ésima actividad y existen $N$ actividades económicas en el área, la probabilidad será $\mathrm{p}_{\mathrm{i}}=n / \mathrm{N}$. El subíndice $i$ corre de $i=1$ hasta el número de actividades económicas, en nuestro caso dicho número es 82 . $\log _{2}$ es el logaritmo base 2, por lo que la información que proporciona el índice se da en bits. Para nuestros cálculos modificamos el índice para obtener números positivos y evitar la multiplicación por -1, esto es:

$$
H=\sum_{i=1}^{n} p_{i} \log _{2}\left(\frac{1}{p_{i}}\right)
$$

Una de las particularidades del índice $\mathrm{H}$ es que cuando las unidades económicas están uniformemente distribuidas, esto es, $p_{i}=p_{j}, i \neq j$, el valor de la entropía es máximo y equivale al $\log _{2} N$. Por ejemplo, si tenemos que en 82 tipos de actividades económicas, todas tienen un número igual de unidades económicas, digamos 25 , la probabilidad $p_{i}=p_{j}, i \neq j$, esto es, la probabilidad $p_{1}=p_{2}=$ $\ldots=p_{82}=25 /(82 \times 25)=0.0121$. La suma de cada una de las 82 probabilidades por el logaritmo base 2 del inverso de la probabilidad nos da 6.3575 y es lo mismo que calcular $\log _{2}(82)=6.3575$.

Por otro lado, Ullman y Dacey (1960) desarrollaron una técnica para medir la estructura de la diversidad industrial en áreas urbanas basada en el principio del número mínimo de puestos de trabajo necesarios para cubrir las necesidades de un sector industrial. Ullman y Dacey definen el empleo «normal» en cada sector industrial, en su caso, para cada categoría de la Clasificación Industrial Estándar Norteamericana. Se considera que dicho porcentaje satisface de manera exacta las necesidades locales. Para estimar este porcentaje, Ullman y Dacey agrupan a las ciudades según el tamaño de su población y elijen el porcentaje mínimo de empleos para cada industria y clase de tamaño de población. Estos mínimos son entonces usados para hacer una regresión sobre la población, las ecuaciones resultantes son usadas para generar estimaciones de los requerimientos mínimos por industria para cada ciudad. El índice de diversidad (D) es calculado por Ullman y Dacey como sigue (Bahl, Firestine y Phares, 1970:415):

$$
D=\frac{\sum_{i=1}^{n} \frac{\left(P_{i}-M_{i}\right)^{2}}{M_{i}}}{\frac{\left(\sum_{i=1}^{n} P_{i}-\sum_{i=1}^{n} M_{i}\right)^{2}}{\sum_{i=1}^{n} M_{i}}}
$$


Donde $P_{i}$ es el porcentaje de empleo en la i-ésima clase industrial. $M_{i}$ es el porcentaje de requerimientos mínimos para la i-ésima clase industrial y $n$ es el número de clases industriales.

Bahl, Firestine y Phares (1970:417) han propusieron una versión simplificada del índice de Ullman-Dacey la cual se define como:

$$
D=\frac{\sum_{i=1}^{n}\left(P_{i}-M_{i}\right)^{2}}{M_{i}}
$$

En donde $P_{i}$ es la proporción de la población total que trabaja en el i-ésimo sector industrial. $M_{i}$ es el porcentaje de requerimientos mínimos para la i-ésima clase industrial. Los mismos Bahl, Firestine y Phares (1970:417) sugieren la aproximación por ojiva en donde se considera como anormal toda desviación de una distribución uniforme del empleo entre las clases industriales, entonces se puede usar una $M_{i}$ igual para todos los casos y entonces la norma para cada clasificación industrial sería 100/(número de clases industriales), en nuestro caso en donde existen 82 clasificaciones, $M_{i}=100 / 82=$ 1.2195. Cabe hacer notar que la formula definida por el Bahl, Firestine y Phares no es adimensional, sino que $D$ queda definido como un porcentaje. Para resolver dicho problema se divide D por $100 \%$, esto con el objeto de dejar adimensional el indicador.

Estudio DE LA DIVERSIDAd DEL COMERCIO MINORISTA EN LA CIUDAd DE SAN LUiS Potosí

El estudio lo desarrollamos dentro del área metropolitana de la ciudad de San Luis Potosí (ZMSLP), México. Dicha zona metropolitana está conformada por las áreas urbanas del municipio de San Luis Potosí y el municipio de Soledad de Graciano Sánchez. El área metropolitana de San Luis Potosí tiene 994,729 habitantes (INEGI, 2010), lo que equivale al $0.9 \%$ del total de la población del país y coloca a la zona metropolitana de San Luis Potosí como la décimo primera en importancia dentro del sistema de metrópolis de México.

Para el estudio, se parte de una unidad espacial censal definida por el Instituto Nacional de Estadística, Geografía e Informática (INEGI). Dicha área es definida oficialmente como área geográfica estadística básica (AGEB). El área metropolitana de la ciudad de San Luis Potosí está dividida en 348 AGEBs. Como fuente de datos para conocer el número y ubicación espacial de las unidades económicas relacionadas con el comercio minorista, se recurrió al Di- 
rectorio Estadístico Nacional de Unidades Económicas (DENUE, 2012) desarrollado por el INEGI.

El primer paso consistió en indagar la estructura espacial del comercio minorista en la ZMSLP con el fin de encontrar aquellos AGEBs que presentaran una mayor concentración de unidades económicas y usar dichos AGEBs como las áreas de estudio. Una vez hecho esto, se procedió a indagar cuál era la estructura espacial dentro de dichos AGEBs. La estructura espacial la identificamos como aquella partición del espacio urbano determinada por manzanas. Es en estas áreas en donde estudiamos la diversidad usando tanto el índice de Shannon-Weaver como el índice de Ullman-Dacey.

Como resultado del primer paso se encontraron que existen en la ZMSLP un total de 19,223 unidades económicas relacionadas con el comercio minorista, repartidas en 348 AGEBs. De éstas, el AGEB 037A y el AGEB 126A son las áreas que contienen el mayor número de unidades económicas. El primer AGEB contiene 1,643 unidades lo que equivale al $8.5366 \%$ del total y el segundo AGEB contiene 637 unidades, esto es, el 3.31\%. El resto de las 346 áreas contienen desde el cero por ciento hasta el 2.12\% de las unidades económicas restantes. Basados en la información anterior se escogieron, de primera intensión, los AGEBs 037A y 126A como áreas de estudio, ya que presentan el mayor número de unidades económicas. Para reforzar nuestra decisión, se calcularon las densidades que presentan el número de unidades de comercio al por menor con respecto al área de cada AGEB y el resultado fue que los AGEBs 037A y 126A presentaron la mayor densidad. Por lo anterior estos dos AGEBs fueron elegidos para realizar el estudio a profundidad de su estructura espacial y la diversidad en cada una de sus manzanas. En el croquis 1 se presenta la ubicación espacial de los AGEBs 037A y 126A dentro de la ciudad. Los croquis de este trabajo se realizaron usando el software IRIS.SCIENCE 4.0.2 proporcionado por el INEGI.

En términos de estructura espacial, la ciudad la podemos clasificar como policentral (Buzai, 2003), al presentar dos centros de actividades económicas correspondientes a los AGEBs 037A y 126A. Cabe aclarar que el AGEB 037A pertenecen al casco histórico de la ZMSLP.

La población total de dicho AGEB es de 2280 habitantes lo que representa el $0.23 \%$ de los habitantes de la ciudad. La población económicamente activa (PEA), del AGEB 037A es de 1071, esto es el 0.25\% del total de la ciudad y el 46.97\% de los habitantes del AGEB. La PEA es definida por el INEGI como todas las personas de 12 y más años que en la semana de referencia realizaron algún tipo de actividad económica, o formaban parte de la población desocupada abierta. 


\section{Croquis 1}

UBICACIÓN DE LOS AGEBS 037A Y 126A DENTRO DE LA ZONA METROPOLITANA DE SAN LUIS POTOSÍ

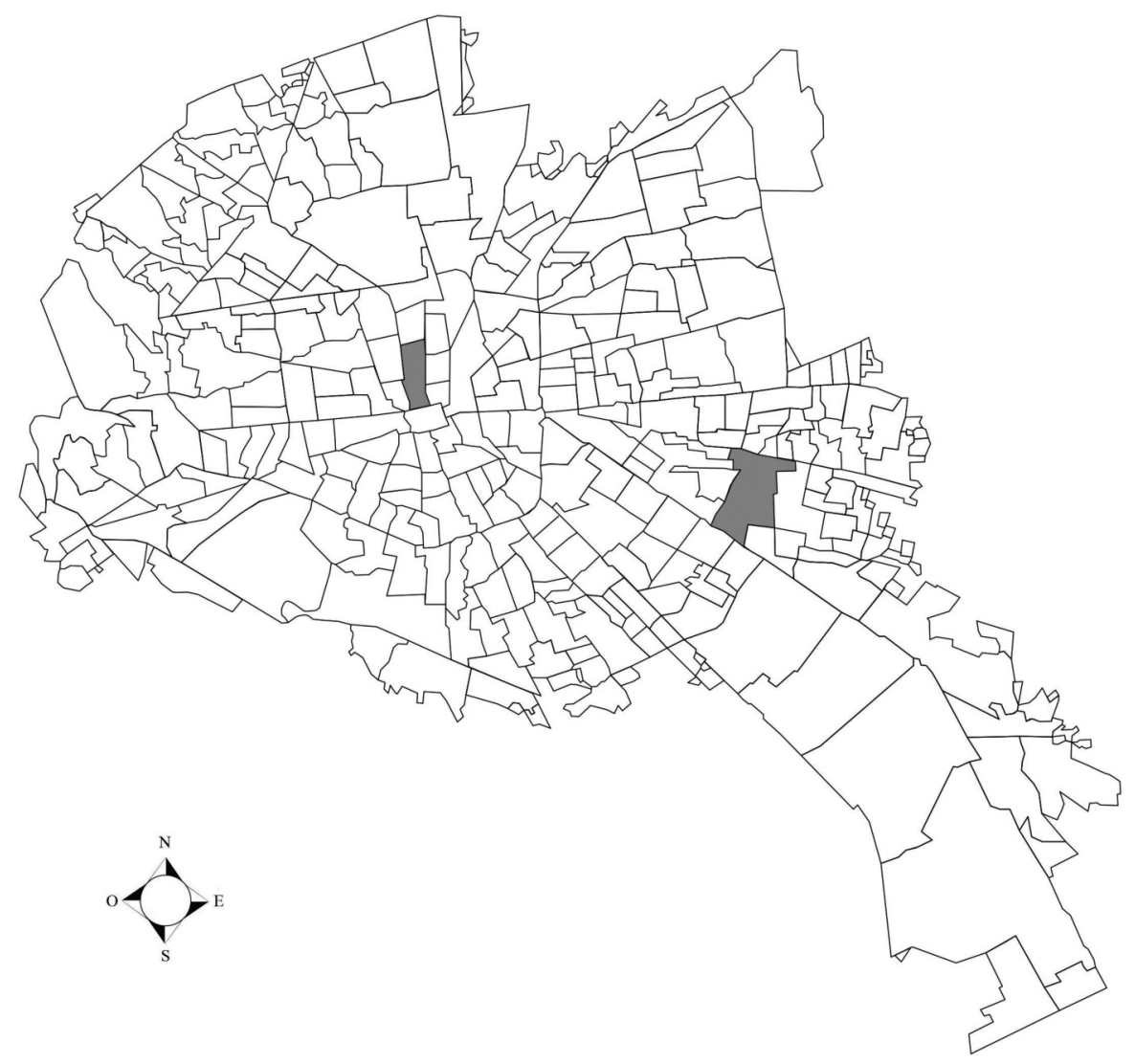

Fuente: realización propia.

El AGEB 126A se encuentra en un centro logístico desarrollado entre los años sesenta y setenta del siglo xx, cuyo fin era concentrar bodegas capaces de abastecer a todos los comercios de la ciudad, evitando la entrada de camiones de carga a la misma (Ayuntamiento de San Luis Potosí, 2003). Dicho AGEB tiene 2.530 habitantes, lo que corresponde al $0.25 \%$ del total de habitantes en la ciudad. LA PEA de este AGEB corresponde al 0.27\% del total de la ciudad y con respecto a los habitantes del AGEB la PEA es de $45.37 \%$. 
Según la clasificación dada por el INEGI (2012a) a través del Directorio Estadístico Nacional de Unidades Económicas (DENUE), existe 82 categorías relacionadas con actividades propias del comercio minorista. En el AGEB 037A existen solo 63 categorías económicas minoristas. Para dicho AGEB, las dos categorías con mayor número de unidades económicas son: ropa, excepto de bebe y lencería con 206 (12\%) unidades y frutas y verduras frescas con 164 (10\%) unidades. Mientras que las actividades que solo tienen una unidad económica son: vinos y licores, cigarros, puros y tabaco, supermercados, ropa de bebe, equipo y material fotográfico, libros, lámparas ornamentales y candiles y aceites y grasas lubricantes, aditivos y asimilares para vehículos de motor. El promedio de actividades económicas en el AGEB 037A es de 26.04, mientras que la desviación estándar es de 36.44, lo que nos indica una gran heterogeneidad en el número de comercios por cuadra. En las tabla 1 del anexo, se

\section{Croquis 2}

\section{DIVISIÓN DEL AGEB 037A POR MANZANA}

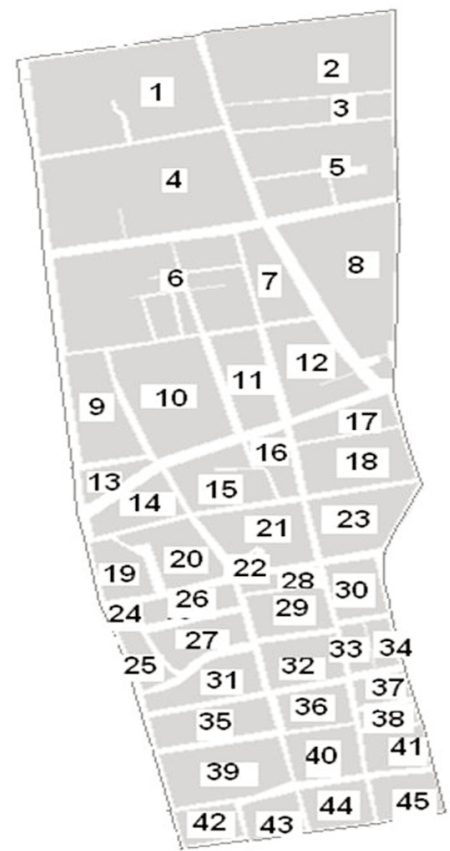

Fuente: elaboración propia. 
muestra el número de unidades existentes para cada una de las 63 actividades económicas para el AGEB 037A.

A continuación, se estudió la estructura espacial del AGEB 037A con respecto a las manzanas que lo conforman. EL AGEB 037A está conformado por 45 manzanas, las cuales fueron numeradas por nosotros. Dicha estructura espacial se muestra en el croquis 2.

Para cada manzana se contabilizaron las unidades económicas de comercio minorista. Cabe hacer notar que la manzana codificada con el número 8 es la que mayor número de unidades económicas presenta, con 510, mientras que las manzanas con menor número de unidades (5) son las codificadas con los números 10 y 22. En la tabla 2 del anexo se muestra el número de comercios al por menor del AGEB 037A para cada una de las 45 manzanas.

El primer cálculo que se realizó fue el encontrar la diversidad para cada manzana usando el índice de Shannon-Weaver. Para ello se encontró cada una de las frecuencias de las 82 actividades económicas para cada manzana y se aplicó la ecuación de Shannon-Weaver. Los resultados para cada una de las manzanas se muestran en la tabla 2 del anexo.

Los resultados obtenidos reflejan que la manzana número 8 , es decir, la que tiene mayor número de unidades económicas, es la que también presenta el mayor índice de diversidad. Recordando que la diversidad máxima sería el $\log _{2}(82)=6.3575$, siendo este número el equivalente al $100 \%$ de diversidad, la manzana número 8 tiene el $64.98 \%$ de diversidad con el índice 4.13156 . Mientras que la manzana número 43 es la menos diversa con solo el 25.21\% de diversidad.

La forma de encontrar las manzanas con mayor diversidad fue determinando la media de del índice de Shannon-Weaver, el cual tienen una media de 2.9004. Las manzanas con el índice mayor a este valor son las que presentan mayor diversidad. Para este caso se tienen 23 manzanas que presentan diversidad según el índice de Shannon-Weaver. En la figura 1 se pueden observar el mapa temático en donde se aprecian por rangos el índice de Shannon-Weber. La media cae en el rango 2.6820-3.07764. Los rangos superiores indican las manzanas presentan diversidad.

A continuación se calculó el índice ojival de Ullman-Dacey sugerido por Bahl, Firestine y Phares (1970:417). Para este cálculo se estimo por manzana el número de empleos para cada una de las 82 actividades económicas relacionadas con el comercio al por menor. El DENUE establece rangos para cada una de las categorías de actividad económica. Para estimar el número de empleados se uso el promedio de la categoría como referencia para los empleos para cada unidad económica contabilizada. Por ejemplo, si en una manzana 
FIGURA 1

MAPA TEMÁTICO DEL ÍNDICE DE DIVERSIDAD DE SHANNON-WEAVER DE COMERCIO AL PORMENOR POR MANZANA PARA EL AGEB 037ª
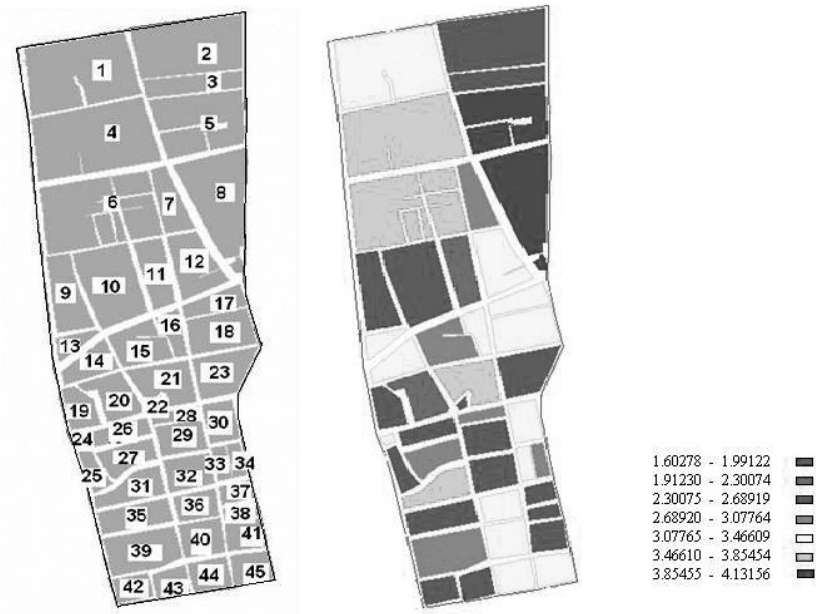

Fuente: elaboración propia.

existen 25 unidades económicas y 20 de ellas caen en el rango de 0 a 5 empleados según el DENUE, el número de empleos estimado será el promedio del rango, esto es, 2.5 redondeado en 3, multiplicado por las 20 unidades, esto es, 60 empleos. Si el resto de las unidades están en el rango de 6 a 10, entonces el promedio es de 8 y el número de empleos será de 40. De esta forma, los empleos para cada unidad económica y cada categoría son estimados.

Sé encontró que la cuadra 8 es la que más alto número de empleos tiene con un estimado de 1.712. Las manzanas con menos empleos (15) son las cuadras 10 y 22. En la tabla 2 del anexo se muestra el número de empleos para cada una de las 45 manzanas en el área de estudio. El índice ojival de UllmanDacey fue calculado para cada una de las 45 manzanas. Para esto se usó la ecuación (4), con $\mathrm{M}=100 / 82=1.2195$. Las $P_{\mathrm{i}}$ se calcularon contabilizando el número de empleos estimados de la i-ésima actividad en la manzana, dividido entre el total de empleos en el AGEB, en el caso del AGEB 037A, el número total de empleos es de 6.145. En la figura 2 se muestra la variación del índice para cada manzana.

Recordando que para el índice de Ullman-Dacey entre más cercano se está del cero, más diversa es el área bajo estudio, encontramos que la manzana 30 
FIGURA 2

\section{MAPA TEMÁTICO DEL ÍNDICE DE ULLMAN-DACEY DEL COMERCIO MINORISTA POR MANZANA DEL AGEB 037A}
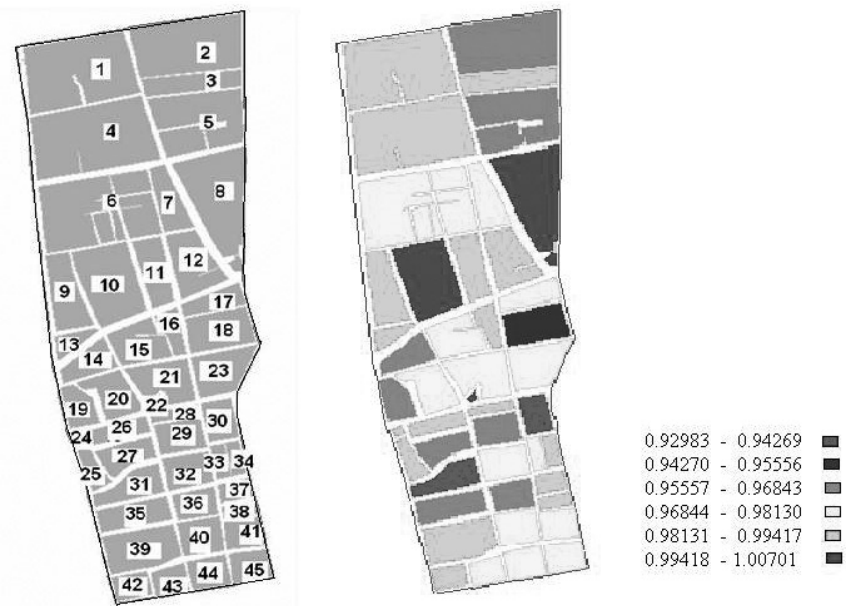

Fuente: elaboración propia.

presenta la mayor diversidad con un índice de 0.9229. Mientras que la manzana menos diversa es la 8 con el índice de 1.007. Usamos la misma técnica que con el índice de Shannon-Weaver para encontrar las manzanas con mayor diversidad. Esto es, calculamos la media y los valores que estén por debajo de la misma son los de mayor diversidad. El índice ojival de Ullman-Dacey tiene una media de 0.9759 para las 45 manzanas con una desviación estándar de 0.0144 , lo que nos indica una homogeneidad grande con respecto al número de empleos ofrecidos por manzana. Encontramos 21 manzanas con una «diversidad» alta en función del criterio anterior. Sin embargo, cabe hacer notar que todos los resultados están alrededor de la media 0.9759 , lo que indica una especialización de las manzanas en cuanto al tipo de empleos ofertados. Además, nótese que no existe consistencia entre lo encontrado usando el índice de Shannon-Weaver. En el apartado dedicado a la discusión de los resultados se abordará este tema.

Para el AGEB 126A se realizaron los mismos cálculos anteriores. Dicho AGEB está conformado por 38 manzanas. Se encontró que en el mencionado AGEB solo existen 56 de las 82 clases económicas minoristas. En el croquis 3 


\section{Croquis 3}

\section{DIVISIÓN DEL AGEB 126A POR MANZANA}

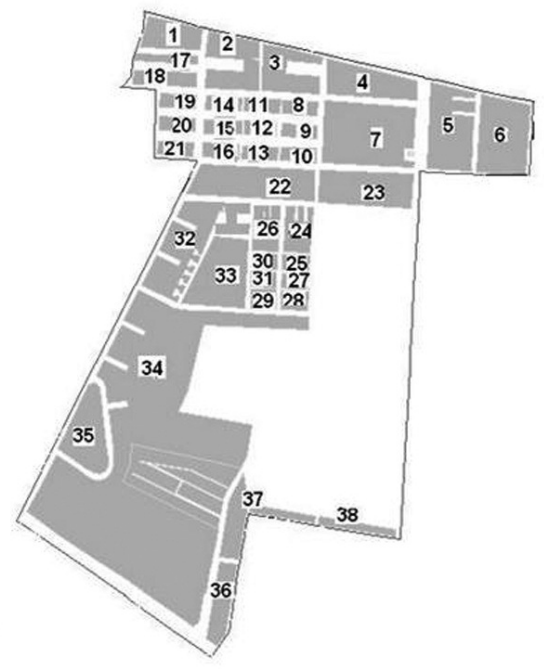

Fuente: elaboración propia.

se muestra la división por manzanas que se realizó para el AGEB 126A. En la tabla 3 del anexo se muestran las clases presentes junto con el número de unidades económicas para cada categoría. En la tabla 4 del anexo se expone el número de empleos y el número de unidades económicas por manzana

Recordando que para el índice de Shannon-Weber se tiene que la máxima entropía está dada por $\log _{2}(82)=6.3575$, encontramos que la manzana codificada con el número 34 presenta la entropía máxima de 4.3402 que corresponde al $68.26 \%$ de la entropía, lo que la hace la manzana más diversa. Encontramos también una manzana con cero unidades económicas que presentan una entropía cero y cuatro manzanas con solo una actividad económica que también dan entropía cero. Nótese que esto equivale a decir que la manzana es altamente especializada. La entropía promedio para el AGEB 126A es de 1.8056, mientras que su desviación estándar es de 1.1515. Para encontrar las manzanas con mayor diversidad usamos en mismo método que para el AGEB 037A, es decir, las manzanas cuya entropía es mayor a 1.8056 son las que presentan mayor diversidad, encontrando 20 manzanas que cumplen con este criterio. En la figura 3 se muestran en forma de mapa temático lo anterior. 
FIGURAS 3

MAPA TEMÁTICO DEL ÍNDICE DE DIVERSIDAD DE SHANNON-WEAVER DEL COMERCIO MINORISTA POR MANZANA PARA EL AGEB 126A
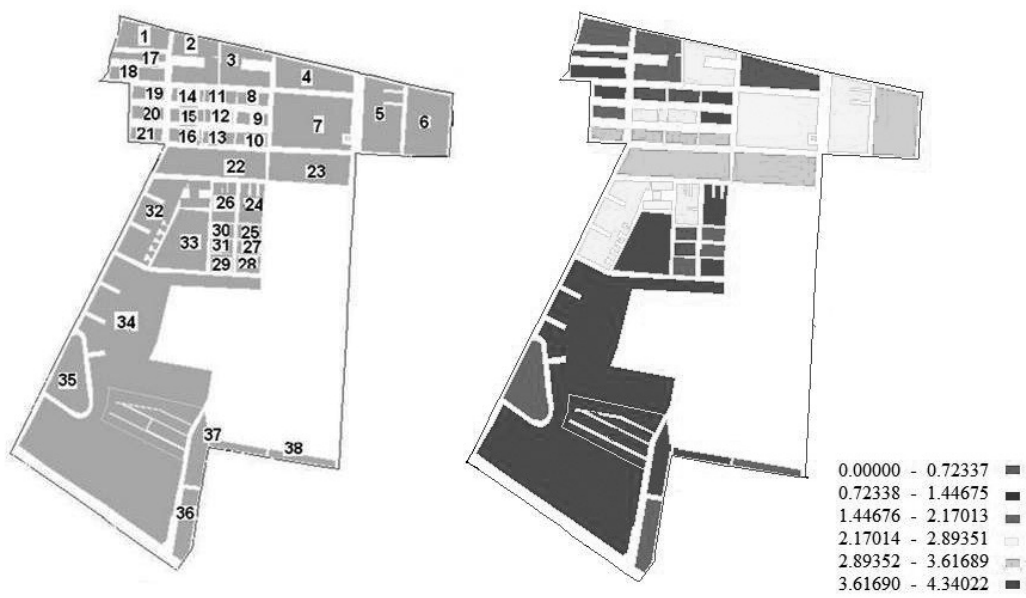

Fuente: elaboración propia.

FIGURAS 4

ÍNDICE DE DIVERSIDAD DE ULLMAN-DACEY DE COMERCIO MINORISTA POR MANZANA PARA EL AGEB 126A
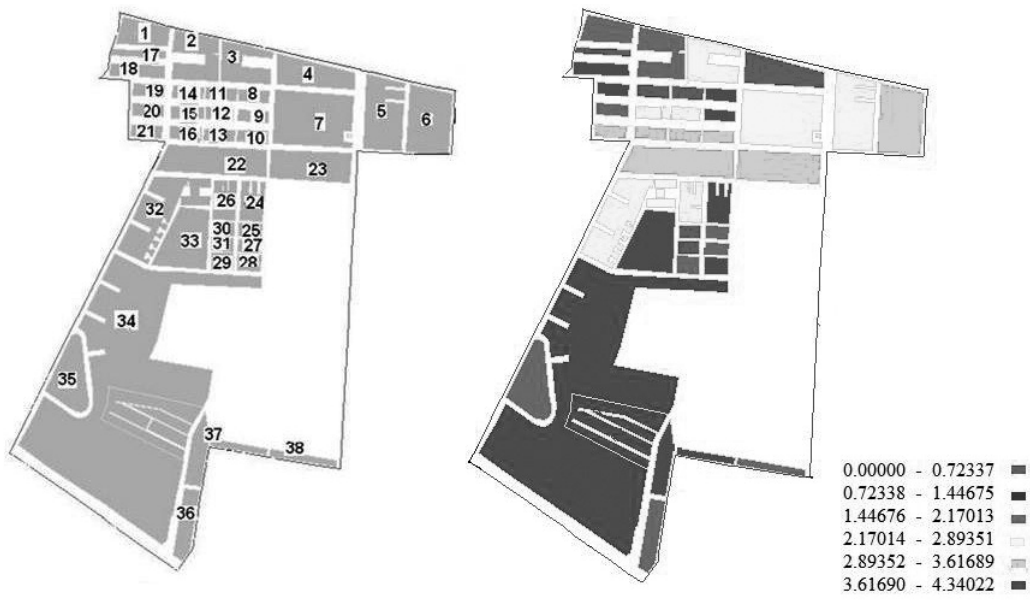

Fuente: elaboración propia.

Estudios Geográficos, Vol. LXXV, 277, pp. 455-478, julio-diciembre 2014 ISSN: 0014-1496, eISSN: 1988-8546, doi: 10.3989/estgeogr.201413 
Para el índice de ojival de Ullman-Dacey se encontraron el número de empleos por manzana, siendo el total de 3368 empleos en el AGEB 126A. Dicha información se expone en la tabla 7 del anexo. Una vez hechos los cálculos pertinentes se encontró que la manzana codificada con el número 34 es la que menor índice tiene con un valor de 0.8619. Mientras que la menos diversa, con el índice 1.1499, es la codificada con el número 18. En promedio el índice tiene un valor de 0.9946 con una desviación estándar de 0.0475 , lo que se interpreta que en general existe homogeneidad en todas las cuadras respecto al índice de Ullman-Dacey. Es notorio que la manzana más diversa según nuestro criterio es la que presenta también el mayor número de empleos con 393, mientras que la que menor diversidad tiene presenta 217 empleos.

Usando nuestro criterio de que los valores por debajo de la media son diversos encontramos 24 manzanas que cumplen dicho criterio. En la figura 4 se puede observar el comportamiento del índice para el AGEB 126A.

\section{DISCUSIÓN Y COMENTARIOS FINALES.}

En este artículo indagamos sobre la diversidad del comercio minorista en los dos principales centros comerciales de la zona metropolitana de San Luis Potosí. Se calcularon para ambas áreas los índices de Shannon-Weaver y el ojival de Ullman-Dacey sugerido por Bahl, Firestine y Phares (1970). El índice de Shannon-Weaver mide la diversidad en función de la variedad de unidades encontradas en cada área de estudio. Las manzanas con mayor diversidad son aquellas que presentan mayor probabilidad de encontrar casi todas o todas las actividades económicas. Dichas manzanas tienen por lo tanto mayor probabilidad de ofertar empleo aún y cuando existan fluctuaciones económicas que obliguen a minimizar empleos en algún sector dado.

Por otra parte el índice ojival de Ullman-Dacey, lo podemos interpretar como un indicador de la «biomasa» por especie, entendiendo por esto al número total de seres de una especie que viven en un lugar determinado, en este caso, los «seres» son los empleos mientras que las especies son las categorías de actividades económicas. Las manzanas que se alejan menos de una distribución uniforme de los empleos, dadas las actividades económicas, son las de mayor diversidad.

En nuestro estudio, encontramos que el índice Shannon-Weaver para el AGEB 037A, índica la presencia de 23 manzanas con alta diversidad. Esto lo podemos interpretar como que el $51.11 \%$ de las manzanas del AGEB son «resistentes» a fluctuaciones del mercado. Además, dicho AGEB contiene 76.83\% 
de las categorías económicas referentes al comercio minorista, lo que se podría interpretar como un área robusta y consolidada que oferta una gran variedad de bienes y por ende la hace atractiva a los compradores potenciales de todas las partes de la ciudad. No obstante, veremos más adelante que esto no es absoluto.

Es interesante notar que la cuadra codificada con el 8 presenta tanto la mayor diversidad de Shannon y al mismo tiempo es la que mayor número de empleos oferta. No obstante, al usar el índice ojival de Ullman-Dacey se observa que es la menos diversa en cuanto al tipo de empleos que oferta. Lo anterior lo interpretamos como sigue: la cuadra número 8 presenta una gran diversidad de comercios minoristas, pero los empleos que ofrecen dichos comercios están concentrados en solo algunos de los sectores económicos.

En general, para el AGEB 037A, solo existe coincidencia entre los índices de Shannon-Weaver y el ojival de Ullman-Dacey en solo 9 de 45 casos. Las cuadras codificadas con los números $6,14,17,18$ 30, 31, 36, 44 y 45 son las que tienen coincidencia entre la información que nos da el índice de Shannon y el índice de Ullman. Además, dichas manzanas ofertan 1503 empleos o sea el $24.46 \%$ de los empleos ofertados en el AGEB. Lo anterior lo podemos interpretar como un 20\% de robustez económica del AGEB.

Para el AGEB 126A observamos que oferta solo el 68.29\% de las actividades económicas minoristas. Encontramos que en dicho AGEB existe una cuadra con cero actividades y cuatro cuadras con solo una actividad. Existen 20 cuadras que presentan una alta diversidad según el índice de Shannon. Sin embargo, ya establecimos que esto, por sí solo, no es contundente para establecer la robustez económica de dicho AGEB. La manzana que presenta el mayor índice de Shannon es la codificada con el número 34 y también tiene el menor índice ojival de Ullman-Dacey. Esto lo interpretamos como un área de gran diversidad de comercios minoristas y donde los empleos que ofrecen dichos comercios están diversificados en varios sectores económicos. En lo general se presentan coincidencias entre los índices de Shannon y de Ullman en 18 (1, 3, 5, 6, 7, 10, $12,13,15,16,20,21,23,26,29,34,35,36)$ de las 38 manzanas $(47.36 \%)$, lo que podemos establecer como que dicho AGEB tiene una robustez del $47.36 \%$.

En resumen, para nuestro estudio los índices de Ullman-Dacey y de Shannon-Weaver los hemos trabajado como complementarios. Siendo su aplicación conjunta útil para estudiar la robustez económica de comercios minoristas en nuestra área de estudio. La observación microscópica que hemos realizado con respecto a las manzanas nos permite tener una mejor comprensión del entorno económico local, pudiendo establecer aquellas unidades espaciales (manzanas) que concentran los empleos en el área, así como las 
unidades que presentan una mejor relación diversidad actividades/diversidad de empleos. Encontramos que el AGEB 037A es el que mayor número de empleos presenta. No obstante, solo 9, de las 45 manzanas que lo conforman, presentan diversidad en ambos índices, lo que nos habla de una concentración de los empleos en solo unas cuantas actividades económicas minoristas. Para el AGEB 126A encontramos que casi la mitad de las manzanas son robustas en cuanto a su relación diversidad actividades económicas/diversidad de empleos. Lo anterior nos permite categorizar al AGEB 126A como más diverso y por ende más adecuado a resistir fluctuaciones económicas que afecten los empleos por actividad económica.

Con respecto a la reflexión en materia de planeación económica podemos adelantar lo siguiente. Se observa que la ciudad de San Luis Potosí es de tipo policentral al presentar dos centros económicos claramente establecidos. Uno en el centro de la ciudad y el otro hacia el nororiente de la misma. La principal área económica, correspondiente al AGEB 037A, es el principal atractor de empleos a nivel minorista. Tomando el hipotético escenario donde la PEA del AGEB cubre en su totalidad la oferta promedio del AGEB, tendríamos 5074 vacantes ofertadas, esto es el $1.20 \%$ del total de la PEA de la ciudad. Lo que hace que dicha área contribuya de forma sustancial al empleo global. En cuanto a los compradores potenciales, estos encuentran en dicha área una buena diversidad de productos que comprar. Además, el área es el lugar donde convergen todas las rutas de transporte público, lo que la hace que esté bien conectada. Sin embargo, los principales usuarios del transporte público es la población con escasos recursos económicos, lo que establece en sí el tipo de precios/calidad de los productos a ofertar. La población del segmento de ingresos medios y altos vive principalmente hacia el sur-poniente de la ciudad y su principal medio de transporte es el automóvil. La falta de suficiente aparcamiento en el AGEB 037A lo hace poco atractivo para el sector de mercado de ingresos medios y altos. Existe la percepción de que los productos ofertados en dicha área económica son de baja calidad. Es patente la carencia de centros comerciales y tiendas de renombre, lo que hace que clientes que buscan más la marca que precios competitivos no se vean motivados a acudir a dicha área.

Dos acciones que se pudieran realizar para mejorar el flujo de compradores con alto nivel de ingresos sería el promover el desarrollo de aparcamientos y la instalación de comercios de renombre. Sin embargo, lo anterior no es sencillo, ya que al ser el centro histórico de la ciudad existe una reglamentación muy rígida en cuanto destrucción y remodelación de predios y el desarrollo de nuevas construcciones, lo que desincentiva la inversión de las cadenas comerciales de renombre que prefieren ubicarse en plazas hechas ex profeso con 
aparcamientos garantizados y ubicadas más hacia el sur poniente de la ciudad, que es donde vive la población de ingresos medios a altos.

En cuanto al AGEB 126A la problemática es también respecto al tipo de clientes que atrae y a su carencia de aparcamientos adecuados. En esta área no hay restricciones respecto a la preservación de predios, pero existe la misma percepción de que el área oferta productos de baja calidad. Además, el área se percibe como insegura con respecto a la criminalidad, lo que desincentiva al comprador no solo de ingresos medios y altos sino también a los de ingresos bajos. Al ubicarse en el nororiente de la ciudad, el área comercial del AGEB $126 \mathrm{~A}$, sirve a la población tanto de dicha área como de las aledañas, dicha población se caracteriza también por sus bajos niveles de ingresos.

En conclusión podemos afirmar que las principales zonas comerciales de la ciudad no son robustas ante cambios cíclicos en la demanda de productos relacionados con el comercio minorista. Existe una especialización en ambas zonas que las hace atractivas para los clientes que buscan productos dentro de los rubros en donde dichas área son especializadas. Su contribución al empleo de la ciudad es sustancial, lo que es un factor de riesgo al ser poco robustas económicamente hablando. Al ser áreas que sirven a estratos de bajos ingresos las hace aún más vulnerables ante procesos inflacionarios y de detrimento del ingreso. La mejoría en el desempeño económico de dichas áreas como centros de comercio es difícil, debido a reglamentaciones que inhiben la inversión en cuanto a comercios de renombre, el desarrollo de aparcamientos y la lejanía con las áreas urbanas habitadas por habitantes con buen nivel económico.

Nuestro estudio lo ubicamos como una investigación dentro del área de la ciencia aplicada. A pesar de lo «añejo» de los índices usados, éstos han sido de utilidad y de fácil uso, permitiéndonos explotar adecuadamente las bases de datos gubernamentales y generar conocimiento pertinente a nivel local. Además, dado el nivel de detalle del trabajo consideramos que éste permite su replicación para otros casos similares.

Fecha de recepción: 23 de noviembre de 2012.

Fecha de aceptación: 26 de junio de 2014.

\section{BiBLIOGRAFÍA}

Attaran, M. and Zwick, M. (1987a): "Entropy and Other Measures of Industrial Diversification". Quarterly Journal of Business and Economics, 26, pp. 17-34.

Attaran, M. and Zwick, M. (1987b): "An Information Theory Approach to Measuring Industrial Diversification". Journal of Economic Studies, 16, pp. 19-30. 
Ayuntamiento de San Luis Potosí (2003): Plan del centro de población estratégico San Luis Potosí-Soledad de Graciano Sánchez.http://www.municipiosoledad.gob.mx/ pdf/trans parencia/Articulo\%2020\%20fraccion\%20III/Plan\%20del\%20Centro\%20de\%20Pobla cion\%20Estrategico\%20SLP\%20-\%20SGS.pdf (Fecha de consulta 31/10/2014).

Bahl, R.W.; Firestine, R. and Phares, D. (1971): "Industrial Diversity in Urban Areas: Alternative Measures and Intermetropolitan Comparisons". Economic Geography, 47/3, pp. 414-425.

Brodsky, H. and Sarfaty, D. E. (1977): "Measuring the urban economic base in a developing country". Land Economics, 53/4, pp. 445-454.

Buzai, G. D. (2003): Mapas sociales urbanos. Buenos Aires, Lugar Editorial.

Dickman, M. (1968): "Some Indices of Diversity". Ecology, 49/6, pp. 1191-1193.

DENUE (2012): Directorio Estadístico Nacional de Unidades Económicas. Disponible en línea, http://www3.inegi.org.mx/sistemas/mapa/denue/default.aspx. (Fecha de consulta: 30/09/2014).

Grossberg, A. J. (1982): "Metropolitan industrial mix and cyclical employment stability". Regional Science Perspectives, 12, pp. 13-35.

Izraeli, O. and Murphy, K. (2003): "The effect of industrial diversity on state unemployment rate and per capita income". Annals of Regional Science, 37/1, pp. 1-14.

INEGI (2010): Censo de Población y Vivienda 2010. Disponible en línea:

http://www.censo2010.org.mx/. (Fecha de consulta 30/09/2014).

Mason, S. and Howard, D. (2010): "Regional industry diversity and its impact on regional unemployment", en: Proceedings of $12^{\text {th }}$ Path to Full Employment/1 $7^{\text {th }} \mathrm{Na}$ tional Unemployment Conference, Newcastle, NSW, 2-3 December, Centre of Full Employment and Equity (CofFEE), Newcastle, NSW, Australia, University of Newcastle. http://epubs.scu.edu.au/cgi/viewcontent.cgi? article=1499\&context= comm_pubs. (Fecha de consulta: 30/09/2014).

Nissan, E. and Carter, G. (2010): "Shannon Measure of Industrial Specialization at the State Level". Southwestern Economic Review, 36/1, pp. 29-46.

Shannon, C. E. (1948): "A Mathematical Theory of Communication". The Bell System Technical Journal, 27, pp. 379-423 y 623-656.

Simon, C. J. (1988): "Frictional Unemployment and the Role of Industrial Diversity". The Quarterly Journal of Economics, 103/(4), pp. 715-728.

Trendle, B. and Shorney, G. (2003): "The effect of industrial diversification on regional economic performance". Australasian Journal of Regional Studies, 9/3, pp, 355-369.

Ullman, E. L. and Dacey, M. F. (1960): "The Minimum Requirements Approach to the Urban Economic Base”. Papers in Regional Science, 6/1, pp. 175-194. 


\section{RESUMEN}

Los índices de Shannon-Weaver y de Ullman-Dacey son usados para medir la diversidad comercial minorista de los dos principales centros de actividad económica de la zona metropolitana de San Luis Potosí, México. Se muestra la forma en que se realizó la definición del área de estudio, el procedimiento de obtención de los datos a través del uso de bases de datos gubernamentales, el modo en que se trabajaron los datos para usarlos en los cálculos de los índices. Por último, se realiza una discusión sobre los resultados y se hacen reflexiones en materia de planeación urbana y económica para el área de estudio.

Palabras Clave: índice de Shannon-Weaver; índice de Ullman-Dacey; diversidad; comercio minorista; San Luis Potosí; México.

\section{AbStract}

The diversity indices of Shannon-Weaver and Ullman-Dacey are used to measure retail commercial diversity of the two main centers of economic activity in the metropolitan area of San Luis Potosi, Mexico. We show how was defined the study area, the method of obtaining data using governmental databases, how we worked the data for use in the calculation of the indices. Finally, we discuss the results and made reflections on urban and economic planning for the study area.

KEY WORDS: Shannon-Weaver index; Ullman-Dacey index; diversity; retail commerce; San Luis Potosi; Mexico.

\section{RÉSUMÉ}

Les indices de Shannon-Weaver et d'Ullman-Dacey sont utilisés pour mesurer la diversité du commerce de détail dans les deux principaux centres d'activité économique de la zone métropolitaine de San Luis Potosí, Mexique. On montre la façon dont on a défini la délimitation du milieu d'étude, le procédé d'obtention des données en utilisant des bases de données gouvernementales et comment les données ont été analysées et utilisées pour calculer les indices. Pour finir, les résultats sont soumis à discussion et des éléments de réflexion sont proposés concernant la planification urbaine et économique du milieu d'étude.

Mots ClÉs: indice de Shannon-Weaver; indice d'Ullman-Dacey; diversité; petit commerce; San Luis Potosí; Mexique. 


\section{ANEXOS}

TABla 1

\section{NÚMERO DE UNIDADES DE COMERCIO MINORISTA DEL AGEB 037A, POR CATEGORÍA}

\begin{tabular}{|c|c|}
\hline Actividades económicas de comercio minorista AGEB 037A & Número \\
\hline Abarrotes, ultramarinos y misceláneas & 76 \\
\hline Carnes rojas & 49 \\
\hline Carne de aves & 15 \\
\hline Pescados y mariscos & 11 \\
\hline Frutas y verduras frescas & 164 \\
\hline Semillas y granos alimenticios, especias y chiles secos & 32 \\
\hline Leche, otros productos lácteos y embutidos & 43 \\
\hline Dulces y materias primas para repostería & 33 \\
\hline Paletas de hielo y helados & 9 \\
\hline Otros alimentos & 10 \\
\hline Vinos y licores & 1 \\
\hline Bebidas no alcohólicas y hielo & 3 \\
\hline Cigarros, puros y tabaco & 1 \\
\hline Supermercados & 1 \\
\hline Tiendas departamentales & 4 \\
\hline Blancos & 2 \\
\hline Artículos de mercería y bonetería & 36 \\
\hline Ropa, excepto de bebe y lencería & 206 \\
\hline Ropa de bebe & 1 \\
\hline Lencería & 7 \\
\hline Disfraces, vestimenta regional y vestidos de novia & 13 \\
\hline Bisutería y accesorios para vestir & 76 \\
\hline Ropa de cuero y piel y de otros artículos de estos materiales & 22 \\
\hline Sombreros & 5 \\
\hline Calzado & 64 \\
\hline Farmacias sin minisúper & 16 \\
\hline Farmacias con minisúper & 4 \\
\hline Productos naturistas, medicamentos homeopáticos y de complementos alimenticios & 47 \\
\hline Lentes & 16 \\
\hline Artículos de perfumería y cosméticos & 16 \\
\hline Artículos de joyería y relojes & 44 \\
\hline Discos y casetes & 25 \\
\hline
\end{tabular}


TABLA 1 (continuación)

NÚMERO DE UNIDADES DE COMERCIO MINORISTA DEL AGEB 037A, POR CATEGORÍA

\begin{tabular}{|c|c|}
\hline Actividades económicas de comercio minorista AGEB 037A & Número \\
\hline Juguetes & 26 \\
\hline Bicicletas & 13 \\
\hline Equipo y material fotográfico & 1 \\
\hline Artículos y aparatos deportivos & 16 \\
\hline Instrumentos musicales & 2 \\
\hline Artículos de papelería & 20 \\
\hline Libros & 1 \\
\hline Revistas y periódicos & 4 \\
\hline Mascotas & 7 \\
\hline Regalos & 86 \\
\hline Artículos religiosos & 11 \\
\hline Artículos desechables & 15 \\
\hline Otros artículos de uso personal & 23 \\
\hline Muebles para el hogar & 27 \\
\hline Electrodomésticos menores y aparatos de línea blanca & 16 \\
\hline Cristalería, loza y utensilios de cocina & 18 \\
\hline Mobiliario, equipo y accesorios de computo & 32 \\
\hline Teléfonos y otros aparatos de comunicación & 46 \\
\hline Alfombras, cortinas, tapices y similares & 2 \\
\hline Plantas y flores naturales & 27 \\
\hline Antigüedades y obras de arte & 2 \\
\hline Lámparas ornamentales y candiles & 1 \\
\hline Otros artículos para la decoración de interiores & 16 \\
\hline Artículos usados & 91 \\
\hline Ferreterías y tlapalerías & 33 \\
\hline Pintura & 13 \\
\hline Vidrios y espejos & 6 \\
\hline Artículos para la limpieza & 27 \\
\hline Partes y refacciones nuevas para automóviles, camionetas y camiones & 4 \\
\hline Otros combustibles & 2 \\
\hline Aceites y grasas lubricantes, aditivos y asimilares para vehículos de motor & 1 \\
\hline
\end{tabular}

Fuente: elaboración propia. 
TABLA 2

NÚMERO DE EMPLEOS Y UNIDADES ECONÓMICAS DE COMERCIO MINORISTA POR MANZANA DEL AGEB 037 ${ }^{a}$

\begin{tabular}{|c|c|c|}
\hline Manzana & Empleos & Unidades de comercio minorista \\
\hline 1 & 53 & 13 \\
\hline 2 & 300 & 95 \\
\hline 3 & 24 & 8 \\
\hline 4 & 53 & 17 \\
\hline 5 & 110 & 38 \\
\hline 6 & 92 & 23 \\
\hline 7 & 60 & 20 \\
\hline 8 & 1712 & 510 \\
\hline 9 & 30 & 10 \\
\hline 10 & 15 & 5 \\
\hline 11 & 18 & 6 \\
\hline 12 & 54 & 18 \\
\hline 13 & 56 & 13 \\
\hline 14 & 170 & 27 \\
\hline 15 & 93 & 31 \\
\hline 16 & 39 & 13 \\
\hline 17 & 97 & 18 \\
\hline 18 & 184 & 52 \\
\hline 19 & 167 & 56 \\
\hline 20 & 92 & 18 \\
\hline 21 & 66 & 22 \\
\hline 22 & 15 & 5 \\
\hline 23 & 83 & 25 \\
\hline 24 & 52 & 16 \\
\hline 25 & 47 & 15 \\
\hline 26 & 51 & 15 \\
\hline 27 & 160 & 57 \\
\hline 28 & 48 & 16 \\
\hline 29 & 172 & 44 \\
\hline 30 & 324 & 90 \\
\hline 31 & 204 & 42 \\
\hline 32 & 181 & 27 \\
\hline 33 & 73 & 17 \\
\hline 34 & 33 & 11 \\
\hline 35 & 198 & 31 \\
\hline 36 & 195 & 17 \\
\hline 37 & 48 & 11 \\
\hline 38 & 48 & 14 \\
\hline 39 & 33 & 10 \\
\hline 40 & 77 & 21 \\
\hline 41 & 114 & 38 \\
\hline 42 & 42 & 14 \\
\hline 43 & 225 & 50 \\
\hline 44 & 132 & 9 \\
\hline 45 & 105 & 35 \\
\hline
\end{tabular}

Fuente: elaboración propia. 
TABLA 3

NÚMERO DE UNIDADES DE COMERCIO MINORISTA DEL AGEB 126A

POR CATEGORÍA

\begin{tabular}{|c|c|}
\hline Actividades económicas de comercio minorista AGEB 126A & Número \\
\hline Abarrotes, ultramarinos y misceláneas & 46 \\
\hline Carnes rojas & 11 \\
\hline Carnes de aves & 3 \\
\hline Frutas y verduras frescas & 290 \\
\hline Semillas y granos alimenticios, especias y chiles secos & 30 \\
\hline Leche, otros productos lácteos y embutidos. & 15 \\
\hline Dulces y materias primas para repostería & 21 \\
\hline Paletas de hielo y helados & 5 \\
\hline Otros alimentos & 19 \\
\hline Vinos y licores & 4 \\
\hline Cerveza & 2 \\
\hline Bebidas alcohólicas y hielo & 11 \\
\hline Supermercados & 1 \\
\hline Minisupers & 2 \\
\hline Tiendas departamentales & 4 \\
\hline Telas & 2 \\
\hline Ropa, excepto bebe y lencería & 19 \\
\hline Ropa para bebe & 1 \\
\hline Bisutería y accesorios para vestir & 6 \\
\hline Ropa de cuero y piel para otros articulos de estos materiales & 1 \\
\hline Pañales desechables & 2 \\
\hline Calzado & 13 \\
\hline Farmacias sin minisuper & 5 \\
\hline Productos naturista, medicamentos homeopáticos y de complementos alimenticios & 2 \\
\hline Lentes & 4 \\
\hline Artículos ortopédicos & 1 \\
\hline Artículos de perfumería y cosméticos & 5 \\
\hline Artículos de joyería y relojes & 4 \\
\hline
\end{tabular}

Estudios Geográficos, Vol. LXXV, 277, pp. 455-478, julio-diciembre 2014 ISSN: 0014-1496, eISSN: 1988-8546, doi: 10.3989/estgeogr.201413 
TABLA 3 (continuación)

NÚMERO DE UNIDADES DE COMERCIO MINORISTA DEL AGEB 126A POR CATEGORÍA

\begin{tabular}{|c|c|}
\hline Actividades económicas de comercio minorista AGEB 126A & Número \\
\hline Discos y casetes & 1 \\
\hline Juguetes & 6 \\
\hline Bicicletas & 1 \\
\hline Equipo y material fotográfico & 1 \\
\hline Artículos y aparatos para el deporte & 3 \\
\hline Artículos de papelería & 11 \\
\hline Mascotas & 2 \\
\hline Regalos & 6 \\
\hline Artículos desechables & 24 \\
\hline Muebles para el hogar & 7 \\
\hline Cristalería, loza y utensilios de cocina & 3 \\
\hline Mobiliario, equipo y accesorios para computo & 1 \\
\hline Teléfonos y otros aparatos de comunicación & 6 \\
\hline Plantas y flores naturales & 2 \\
\hline Artículos usados & 1 \\
\hline Ferreterías y tlapalerías & 6 \\
\hline Pintura & 1 \\
\hline Vidrios y espejos & 1 \\
\hline Artículos para la limpieza & 7 \\
\hline Automóviles y camionetas nuevos & 1 \\
\hline Automóviles y camionetas usadas & 1 \\
\hline Partes y refacciones nuevas para automóviles camionetas y camiones & 6 \\
\hline Llantas y cámaras para automóviles, camionetas y camiones & 3 \\
\hline Gasolina y diesel & 1 \\
\hline Gas L.P. en cilindros y para tanques estacionarios & 1 \\
\hline Otros combustibles & 1 \\
\hline Aceites y grasas lubricantes, aditivos y similares para vehículos de motor. & 3 \\
\hline Exclusivamente a través de internet y catálogos impresos, televisión y similares & 1 \\
\hline
\end{tabular}

Fuente: elaboración propia. 
TABLA 4

NÚMERO EMPLEOS Y DE UNIDADES DE COMERCIO MINORISTA PARA CADA UNA DE LAS MANZANAS DEL AGEB $126^{\mathrm{a}}$

\begin{tabular}{|c|c|c|}
\hline Manzana & Empleos & Unidades de comercio minorista \\
\hline 1 & 55 & 4 \\
\hline 2 & 107 & 24 \\
\hline 3 & 227 & 25 \\
\hline 4 & 6 & 2 \\
\hline 5 & 71 & 11 \\
\hline 6 & 60 & 14 \\
\hline 7 & 35 & 20 \\
\hline 8 & 167 & 44 \\
\hline 9 & 137 & 33 \\
\hline 10 & 156 & 30 \\
\hline 11 & 162 & 44 \\
\hline 12 & 76 & 12 \\
\hline 13 & 132 & 16 \\
\hline 14 & 170 & 39 \\
\hline 15 & 42 & 8 \\
\hline 16 & 106 & 20 \\
\hline 17 & 14 & 4 \\
\hline 18 & 217 & 48 \\
\hline 19 & 115 & 29 \\
\hline 20 & 68 & 19 \\
\hline 21 & 54 & 18 \\
\hline 22 & 291 & 24 \\
\hline 23 & 34 & 8 \\
\hline 24 & 44 & 2 \\
\hline 25 & 9 & 3 \\
\hline 26 & 36 & 12 \\
\hline 27 & 14 & 3 \\
\hline 28 & 24 & 2 \\
\hline 29 & 12 & 4 \\
\hline 30 & 6 & 2 \\
\hline 31 & 0 & 0 \\
\hline 32 & 241 & 12 \\
\hline 33 & 6 & 2 \\
\hline 34 & 393 & 84 \\
\hline 35 & 48 & 4 \\
\hline 36 & 12 & 4 \\
\hline 37 & 6 & 2 \\
\hline 38 & 15 & 5 \\
\hline
\end{tabular}

Fuente: elaboración propia.

Estudios Geográficos, Vol. LXXV, 277, pp. 455-478, julio-diciembre 2014 ISSN: 0014-1496, eISSN: 1988-8546, doi: 10.3989/estgeogr.201413 